\title{
THE NASA-UC-UH ETA-EARTH PROGRAM. IV. A LOW-MASS PLANET ORBITING AN M DWARF 3.6 PC FROM EARTH*
}

\author{
Andrew W. Howard ${ }^{1}$, Geoffrey W. Marcy ${ }^{2}$, Debra A. Fischer ${ }^{3}$, Howard IsaAcson ${ }^{2}$, Philip S. Muirhead $^{4,11}$, \\ Gregory W. Henry ${ }^{5}$, Tabetha S. Boyajian ${ }^{3}$, Kaspar von Braun ${ }^{6,7}$, Juliette C. Becker ${ }^{4}$, \\ JASON T. Wright ${ }^{8,9}$, AND JOHN ASHER JOHNSON ${ }^{10,12}$ \\ ${ }^{1}$ Institute for Astronomy, University of Hawaii, 2680 Woodlawn Drive, Honolulu, HI 96822, USA \\ ${ }^{2}$ Department of Astronomy, University of California, Berkeley, CA 94720-3411, USA \\ ${ }^{3}$ Department of Astronomy, Yale University, New Haven, CT 06511, USA \\ ${ }^{4}$ Department of Astrophysics, California Institute of Technology, MC 249-17, Pasadena, CA 91125, USA \\ ${ }^{5}$ Center of Excellence in Information Systems, Tennessee State University, 3500 John A. Merritt Boulevard, Box 9501, Nashville, TN 37209 , USA \\ ${ }^{6}$ NASA Exoplanet Science Institute, California Institute of Technology, Pasadena, CA 91125, USA \\ ${ }^{7}$ Max-Planck-Institute for Astronomy, Königstuhl 17, D-69117 Heidelberg, Germany \\ ${ }^{8}$ Department of Astronomy and Astrophysics, The Pennsylvania State University, University Park, PA 16802, USA \\ ${ }^{9}$ Center for Exoplanets and Habitable Worlds, The Pennsylvania State University, University Park, PA 16802, USA \\ ${ }^{10}$ Center for Planetary Astronomy, California Institute of Technology, 1200 East California Boulevard, Pasadena, CA 91125, USA \\ Received 2013 June 13; accepted 2014 August 13; published 2014 September 23
}

\begin{abstract}
We report the discovery of a low-mass planet orbiting Gl 15 A based on radial velocities from the Eta-Earth Survey using HIRES at Keck Observatory. Gl $15 \mathrm{Ab}$ is a planet with minimum mass $M \sin i=5.35 \pm 0.75 M_{\oplus}$, orbital period $P=11.4433 \pm 0.0016$ days, and an orbit that is consistent with circular. We characterize the host star using a variety of techniques. Photometric observations at Fairborn Observatory show no evidence for rotational modulation of spots at the orbital period to a limit of $\sim 0.1 \mathrm{mmag}$, thus supporting the existence of the planet. We detect a second RV signal with a period of 44 days that we attribute to rotational modulation of stellar surface features, as confirmed by optical photometry and the Ca II H \& K activity indicator. Using infrared spectroscopy from Palomar-TripleSpec, we measure an M2 V spectral type and a sub-solar metallicity $([\mathrm{M} / \mathrm{H}]=-0.22,[\mathrm{Fe} / \mathrm{H}]=-0.32)$. We measure a stellar radius of $0.3863 \pm 0.0021 R_{\odot}$ based on interferometry from CHARA.
\end{abstract}

Key words: planetary systems - stars: individual (Gliese 15 A) - techniques: radial velocities

Online-only material: color figures

\section{INTRODUCTION}

The nearest and brightest stars are among the best studied and hold a special place in the popular imagination. The discovery of planets orbiting these stars tells us that the solar neighborhood is potentially rich with solar systems. Within 7 pc, we know of gas giant planets orbiting $\epsilon$ Eridani (Hatzes et al. 2000), Gl 876 (Marcy et al. 1998; Delfosse et al. 1998), and Gl 832 (Bailey et al. 2009), intermediate mass planets commonly called Neptunes and super-Earths orbiting Gl 674 (Bonfils et al. 2007), Gl 876 (Rivera et al. 2010), HD 20794 (Pepe et al. 2011), and Gl 581 (Mayor et al. 2009), and an approximately Earth-mass $\left(M_{\oplus}\right)$ planet orbiting $\alpha$ Centauri B (Dumusque et al. 2012). To this list, we add a $5 M_{\oplus}$ planet orbiting the star Gl $15 \mathrm{~A}$, which at $3.6 \mathrm{pc}$ is a member of the 16th closest stellar system cataloged by RECONS ${ }^{13}$ (e.g., Jao et al. 2005; Henry et al. 2006).

This new planet was discovered as part of the Eta-Earth Survey, a census of planets orbiting the nearest stars. Our target list is composed of $232 \mathrm{G}, \mathrm{K}$, and M stars suitable for high-precision Doppler observations at the Keck Observatory. These stars are nearby (within $25 \mathrm{pc}$ ), bright $(V<11)$, and

\footnotetext{
* Based on observations obtained at the W. M. Keck Observatory, which is operated jointly by the University of California and the California Institute of Technology. Keck time was granted for this project by the University of Hawaii, the University of California, and NASA.

${ }^{11}$ Current address: Department of Astronomy, Boston University, 725

Commonwealth Avenue, Boston, MA 02215, USA.

12 Current address: Harvard-Smithsonian Center for Astrophysics, 60 Garden

Street, Cambridge, MA 02138, USA.

13 http://www.astro.gsu.edu/RECONS/
}

have low chromospheric activity $\left(\log R_{\mathrm{HK}}^{\prime}<-4.7\right)$. Each star is searched for planets-particularly close-in, low-mass planets-using a minimum of 20 radial velocity (RV) measurements of $\sim 1 \mathrm{~m} \mathrm{~s}^{-1}$ precision from the HIRES spectrometer. This survey has detected several low-mass planets (Howard et al. 2009, 2011a, 2011b) and showed that for G and $\mathrm{K}$ dwarfs the planet mass function rises steeply with decreasing planet mass: small planets are common (Howard et al. 2010b). The $66 \mathrm{M}$ dwarfs in the Eta-Earth Survey sample were excluded from that statistical study of planet occurrence because many stars lacked sufficient measurements to confidently detect or exclude the presence of low-mass planets.

In this paper, we characterize the planet host star Gl 15 A using a variety of observational techniques (Section 2), describe Doppler measurements of the star (Section 3), announce the existence of the close-in, super-Earth Gl $15 \mathrm{Ab}$ (Section 4), and discuss this new planet in the context of the properties of known small planets (Section 5).

\section{STELLAR CHARACTERIZATION}

Gl 15 A (also known as HD 1326 A, HIP 1475, GX Andromedae, and Groombridge 34) is a nearby, cool dwarf of type M1 (Reid et al. 1995) or M2 (this work). The other member of this binary star system, Gl $15 \mathrm{~B}$, is fainter and has a spectral type of M3.5 dwarf (Reid et al. 1995). Lippincott (1972) measured a small astrometric segment of their orbit, giving an $A B$ separation of $146 \mathrm{AU}$ and an orbital period of $2600 \mathrm{yr}$. Assuming an edge-on viewing geometry of $\mathrm{AB}$ and a circular orbit, the 
Table 1

Adopted Stellar Properties of Gl 15 A

\begin{tabular}{lcc}
\hline \hline Parameter & G1 15 A & Source \\
\hline Spectral type & M2 V & TripleSpec spectra and \\
& & IRTF Spectral Library \\
$B-V(\mathrm{mag})$ & 1.55 & Leggett (1992) \\
$V(\mathrm{mag})$ & 8.08 & Leggett (1992) \\
$J$ (mag) & 4.82 & Leggett (1992) \\
$H(\mathrm{mag})$ & 4.25 & Leggett (1992) \\
$K(\mathrm{mag})$ & 4.03 & Leggett (1992) \\
Distance (pc) & $3.587 \pm 0.010$ & van Leeuwen (2007) \\
$T_{\text {eff }}(\mathrm{K})$ & $3567 \pm 11$ & SED fit \\
$\log g(\mathrm{cgs})$ & $4.90 \pm 0.17$ & computed from $M_{\star}$ and $R_{\star}$ \\
{$[\mathrm{Fe} / \mathrm{H}](\mathrm{dex})$} & $-0.32 \pm 0.17^{\mathrm{a}}$ & TripleSpec spectra calibrated by \\
& & Rojas-Ayala et al. (2012) \\
{$[\mathrm{M} / \mathrm{H}](\mathrm{dex})$} & $-0.22 \pm 0.12^{\mathrm{a}}$ & TripleSpec spectra calibrated by \\
& & Rojas-Ayala et al. (2012) \\
$v \sin i\left(\mathrm{~km} \mathrm{~s}{ }^{-1}\right)$ & $1.45 \pm 0.6$ & Houdebine (2010) \\
$L_{\star}\left(L_{\odot}\right)$ & $0.02173 \pm 0.00021$ & CHARA interferometry and SED fit \\
$M_{\star}\left(M_{\odot}\right)$ & $0.375 \pm 0.057$ & TripleSpec parameters with \\
$R_{\star}\left(R_{\odot}\right)$ & $0.3863 \pm 0.0021$ & Dartmouth isochrones \\
$S_{\mathrm{HK}}$ & $0.527 \pm 0.038$ & CHARA interferometry \\
& & Ca II H \& K (HIRES)
\end{tabular}

Note. ${ }^{\text {a }}$ The uncertainties for $[\mathrm{M} / \mathrm{H}]$ and $[\mathrm{Fe} / \mathrm{H}]$ from TripleSpec are dominated by systematic errors. For comparison with other stars using the same calibration, the photon-limited errors on these measurements are 0.02 dex.

maximum RV acceleration of $\mathrm{A}$ due to $\mathrm{B}$ is $\sim 2 \mathrm{~m} \mathrm{~s}^{-1} \mathrm{yr}^{-1}$. Based on an imaging search for companions at $10 \mu \mathrm{m}$ with MIRLIN at Palomar, van Buren et al. (1998) ruled out additional companions to A having projected separations of 9-36 AU with $T_{\text {eff }}>1800 \mathrm{~K}\left(M_{\star}>0.084 M_{\odot}\right)$. Gautier et al. (2007) found no infrared excess for Gl $15 \mathrm{~A}$ at 24, 70, or $160 \mu \mathrm{m}$.

Since G1 $15 \mathrm{~A}$ is a bright nearby star, we undertook a significant campaign to characterize it using a combination of high-resolution optical spectroscopy, near infrared (IR) spectroscopy, long-baseline optical/infrared interferometry, and high-cadence, broadband optical photometry. In the subsections that follow, we describe these measurements, which are summarized in Table 1.

\subsection{HIRES Optical Spectroscopy}

We observed Gl 15 A with the HIRES echelle spectrometer (Vogt et al. 1994) on the $10 \mathrm{~m}$ Keck I telescope using standard procedures. The observations reported here span 15 yr (1997 January through 2011 December) and were made with an iodine cell mounted directly in front of the spectrometer entrance slit to measure precise relative RVs. The dense set of molecular absorption lines imprinted on the stellar spectra provide a robust wavelength fiducial against which Doppler shifts are measured, as well as strong constraints on the shape of the spectrometer instrumental profile at the time of each observation (Marcy \& Butler 1992; Valenti et al. 1995).

With the iodine cell removed, we also measured a "template" spectrum of Gl15 A that was used in the Doppler analysis (Section 3). For stars with $T_{\text {eff }} \gtrsim 4800 \mathrm{~K}$, we typically measure stellar parameters using the Spectroscopy Made Easy (SME; Valenti \& Piskunov 1996; Valenti \& Fischer 2005) LTE spectral synthesis tool. However, below this temperature SME is unreliable. For Gl 15 A, we measured stellar parameters using the techniques below.

Measurements of the cores of the $\mathrm{Ca}$ II $\mathrm{H} \& \mathrm{~K}$ lines of each HIRES spectrum (outside the $\sim 5000-6200 \AA$ region affected by

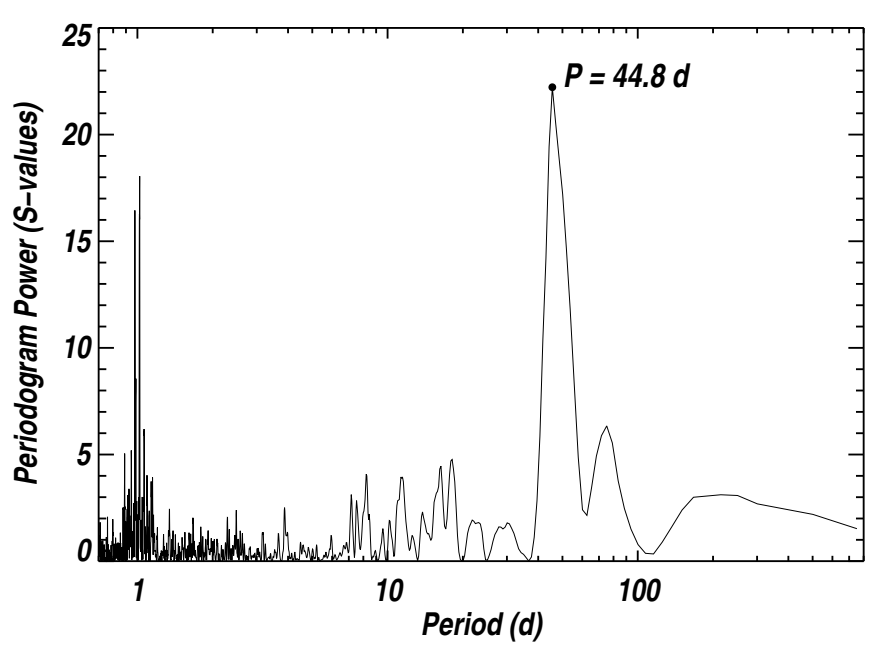

Figure 1. Lomb-Scargle periodogram of $\mathrm{S}_{\mathrm{HK}}$ values from 59 measurements during the 2011 observing season (BJD > 2,455,500). We interpret the prominent peak near 44 days as due to modulation of stellar surface features (spots and plagues) at the stellar rotation period. The peaks near one day are aliases of our observing cadence and longer period signals.

iodine lines) show modest levels of chromospheric activity, as quantified by the $S_{\mathrm{HK}}$ and $\log R_{\mathrm{HK}}^{\prime}$ indices (Isaacson \& Fischer 2010 ). The $S_{\mathrm{HK}}$ values (Table 3 ) are computed to a precision of 0.001 and carry single measurement uncertainties of $\sim 0.002$. Values in Table 3 that are reported to a precision less than 0.001 are the result of binning multiple measurements in a two hour timespan. These measurements are variable on both short and long timescales. Over our $15 \mathrm{yr}$ baseline, we detect a $9 \pm 2.5 \mathrm{yr}$ cycle with a semi-amplitude of $\sim 0.05$ (in the dimensionless units of $S_{\mathrm{HK}}$ ), which is a $\sim 10 \%$ fractional change. This variation may be a magnetic activity cycle analogous to the solar cycle. We are unable to check for a similar variation in the optical photometry from Fairborn Observatory below (Section 2.4), which spans only four years.

To measure variability on shorter timescales, we examined $59 S_{\mathrm{HK}}$ measurements from the 2011 observing season (BJD > 2,455,500). A Lomb-Scargle periodogram of these data (Figure 1) shows a clear periodicity near 44 days that we interpret as the rotation period. As shown below, we also detect $\sim 44$ day modulations in optical photometry and the RV time series, which is consistent with the rotational modulation of stellar surface features.

\subsection{Palomar-TripleSpec Infrared Spectrscopy}

We observed Gl 15 A with the TripleSpec Spectrograph on the Palomar Observatory 200 inch Hale Telescope on UT 2012 February 12. TripleSpec is a near-infrared, long-slit spectrograph covering 1.0-2.5 $\mu \mathrm{m}$ simultaneously with a resolving power of 2700 (Wilson et al. 2004; Herter et al. 2008). The TripleSpec detector does not have a shutter and instead records differences between multiple nondestructive readouts for individual exposures (Fowler \& Gatley 1990). The readout time sets the minimum exposure time of the detector to $3.8 \mathrm{~s}$. To prevent saturation of this bright target, we guided with only a wing of the seeing-limited image of G1 15 A on the slit. Two positions along the slit (A and B) were used. Exposures were taken in an ABBA pattern with the minimum exposure time.

We reduced the data using the SpexTool program, modified for Palomar-TripleSpec (Cushing et al. 2004; M. Cushing 2011, private communication). The spectra were cleansed of telluric 


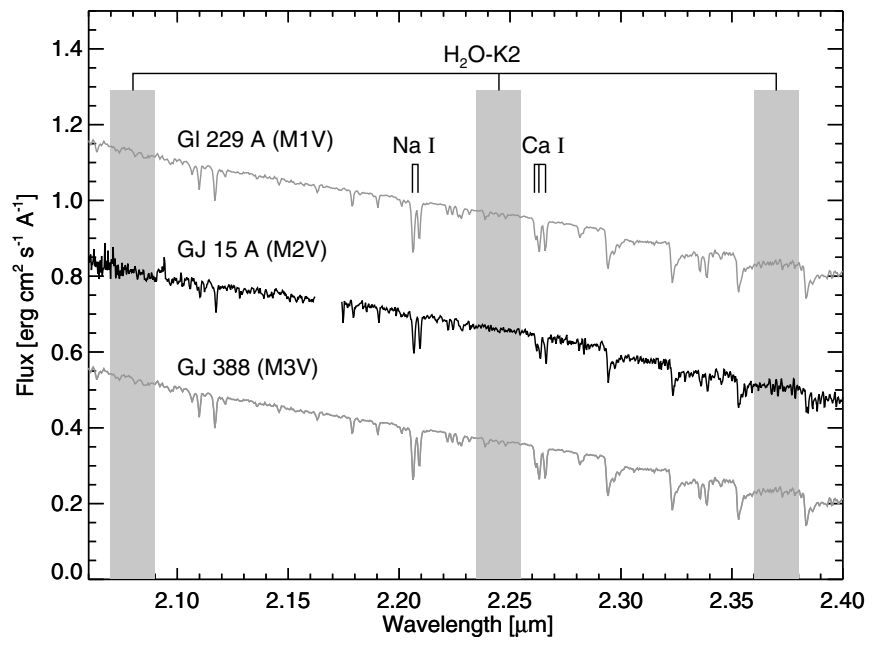

Figure 2. Palomar-TripleSpec $K$-band spectrum of Gl 15 A, with comparison stars drawn from the IRTF Spectral Library (KHM spectral types; Cushing et al. 2005; Rayner et al. 2009). The templates are adjusted to the same scale as the G1 15 A spectrum using a ratio of the median flux in $K$ band, and then artificially offset. We used spectral indices defined in Rojas-Ayala et al. (2012) to measure the $T_{\text {eff }},[\mathrm{M} / \mathrm{H}]$, and $[\mathrm{Fe} / \mathrm{H}]$ : the $\mathrm{H}_{2} \mathrm{O}-\mathrm{K} 2$ index (gray shaded regions) and the equivalent widths of the $\mathrm{Na}$ I doublet and $\mathrm{Ca}$ I triplet features (indicated).

absorption lines by comparison with spectra of a nearby A0V star (BD+43 61) and were flux calibrated using the xtellcor package (Vacca et al. 2003). The brightness of Gl 15 A resulted in a discontinuity in the reduced spectrum at the location of a quadrant boundary in the detector. We manually adjusted the normalization of the spectrum to remove the discontinuity. Figure 2 shows the reduced $K$-band spectrum of G1 $15 \mathrm{~A}$, with comparison spectra of bracketing spectral types.

Moderate resolution $K$-band spectra of $\mathrm{M}$ dwarfs are sensitive to stellar temperature (Covey et al. 2010) and metallicity (Rojas-Ayala et al. 2010, 2012). For Gl 15 A, we measured spectral indices and used the Rojas-Ayala et al. (2012) calibrations to estimate $T_{\text {eff }},[\mathrm{M} / \mathrm{H}]$, and $[\mathrm{Fe} / \mathrm{H}]$. We measured the equivalent widths of $3.430 \pm 0.098 \AA$ and $3.148 \pm 0.103 \AA$ for the $K$-band $\mathrm{Na}$ I and $\mathrm{Ca}$ I absorption features, respectively. For the $\mathrm{H}_{2} \mathrm{O}-\mathrm{K} 2$ index, we measured a value of $0.95434 \pm 0.0030$. From these measurements, we infer $[\mathrm{Fe} / \mathrm{H}]=-0.32 \pm 0.17$ and $[\mathrm{M} / \mathrm{H}]=-0.22 \pm 0.12$ using the Rojas-Ayala et al. (2012) calibration. These metallicity estimates are consistent with the broadband photometric calibration by Johnson \& Apps (2009), which predicts $[\mathrm{Fe} / \mathrm{H}]=-0.25 \pm 0.07$.

Following Rojas-Ayala et al. (2012), we interpolated [M/H] and the $\mathrm{H}_{2} \mathrm{O}-\mathrm{K} 2$ index on a surface of $[\mathrm{M} / \mathrm{H}], \mathrm{H}_{2} \mathrm{O}-\mathrm{K} 2$, and $T_{\text {eff }}$ calculated using synthetic spectra (Allard et al. 2012). The resulting temperature $T_{\text {eff }}=3568 \pm 52 \mathrm{~K}$ is consistent with the temperature estimate of $3567 \pm 11 \mathrm{~K}$ from fitting the spectral energy distribution and CHARA interferometry (Section 2.3).

We estimated uncertainties using a Monte Carlo approach combined with calibration uncertainties from Rojas-Ayala et al. (2012). SpexTool reports per-channel uncertainties based on the photon noise and read noise in both the target and calibration data. We created 1000 instances of the data, each with random, normally distributed noise added based on the reported per-channel uncertainties. For each instance, we calculated the spectral indices and report the standard deviation in the distribution of values as the uncertainty. We also calculated $T_{\text {eff }}$, $[\mathrm{M} / \mathrm{H}]$, and $[\mathrm{Fe} / \mathrm{H}]$ for each iteration; however, these are also subject to uncertainties in the calibration relations. Rojas-Ayala et al. (2012) calculate an uncertainty of 0.17 in the $[\mathrm{Fe} / \mathrm{H}]$ relation and 0.12 in the $[\mathrm{M} / \mathrm{H}]$ relation, which dominate over the Monte Carlo uncertainty estimations. We estimate the uncertainty in the effective temperature calculation to be $50 \mathrm{~K}$, which also dominates over the Monte Carlo estimation.

We interpolated the measured $[\mathrm{M} / \mathrm{H}]$ and $T_{\text {eff }}$ on stellar evolutionary models to determine $M_{\star}, R_{\star}$, and $L_{\star}$. We explored $5 \mathrm{Gyr}$ isochrones from two sets of evolutionary models: the Dartmouth evolutionary models (e.g., Dotter et al. 2008; Feiden et al. 2011) and the BCAH evolutionary models (Baraffe et al. 1998). The age assumption does not change stellar parameters by more $1 \%$ for ages over $1 \mathrm{Gyr}$. To estimate uncertainties, we again used a Monte Carlo approach, interpolating 1000 iterations of $T_{\text {eff }}$ and $[\mathrm{M} / \mathrm{H}]$ onto the isochrones, with each iteration containing normally distributed noise based on the calculations described above. For the Dartmouth models, respectively, we estimate $M_{\star}=0.375 \pm 0.057 M_{\odot}, R_{\star}=0.363 \pm 0.052 R_{\odot}$, and $L_{\star}=0.0191 \pm 0.0040 L_{\odot}$. For the BCAH models, respectively, we estimate $M_{\star}=0.358 \pm 0.065 M_{\odot}, R_{\star}=0.342 \pm$ $0.057 R_{\odot}$, and $L_{\star}=0.0170 \pm 0.0045 L_{\odot}$. These radius and luminosity estimates are consistent with those from CHARA interferometry and spectral energy distribution (SED) modeling (Section 2.3).

For comparison, we estimate the mass of Gl 15 A using the absolute $K$-band mass-luminosity calibration of Delfosse et al. (2000). We find $M_{\star}=0.404 \pm 0.033 M_{\odot}$ based on $K=4.03$ mag (Leggett 1992) and a distance of $3.587 \pm 0.010 \mathrm{pc}$ (van Leeuwen 2007). The mass uncertainty comes from 0.18 mag rms errors in fitting the $K$-band photometry in Delfosse et al. (2000; see Section 3.6 of Johnson et al. 2012).

\subsection{CHARA Interferometry}

\subsubsection{Interferometry and Stellar Diameter}

We measured the angular diameter of G1 15 A using visibility interferometry. We observed Gl 15 A over seven nights between 2008 September and 2011 August using the Georgia State University Center for High Angular Resolution Astronomy (CHARA) Array (ten Brummelaar et al. 2005) as part of a survey of $\mathrm{K}$ and $\mathrm{M}$ dwarfs (Boyajian et al. 2012). We obtained 40 interferometric observations in $H$ and $K$ bands in single baseline mode, using 6 of CHARA's longest available baselines $\left(217<B_{\max }<331 \mathrm{~m}\right)$. Our observational strategy is described in von Braun et al. (2011a, 2011b), including the use of calibrator stars to remove the influence of atmospheric and instrumental systematics. Our calibrator stars were chosen to be near-pointlike sources of similar brightness as G1 $15 \mathrm{~A}$ and are separated by small angular distances. These stars are HD 6920, HD 905, and HD 3765.

We measured a limb-darkening-corrected (Claret 2000) angular diameter of $1.005 \pm 0.005$ milliarcseconds (mas). Using the van Leeuwen (2007) distance, we convert the angular diameter to a physical diameter of $R_{\star}=0.3846 \pm 0.0023 R_{\odot}$, in agreement with previous CHARA measurements of $0.379 \pm 0.006 R_{\star}$ (Berger et al. 2006) and $0.393 \pm 0.023 R_{\star}$ (van Belle \& von Braun 2009).

With this well-measured stellar radius and rotation period, we can make a comparison with the spectroscopic broadening kernel, $v \sin i$. For an equatorial viewing geometry $(\sin i=1)$, we the expect $v \sin i \approx V_{\text {rot }} \approx 2 \pi R_{\star} / P_{\text {rot }}$. For Gl $15 \mathrm{~A}$, we find $2 \pi R_{\star} / P_{\text {rot }}=0.44 \mathrm{~km} \mathrm{~s}^{-1}$, which is inconsistent at the $1.7 \sigma$ level with the measured $v \sin i=1.45 \pm 0.6 \mathrm{~km} \mathrm{~s}^{-1}$ (Houdebine 2010). 


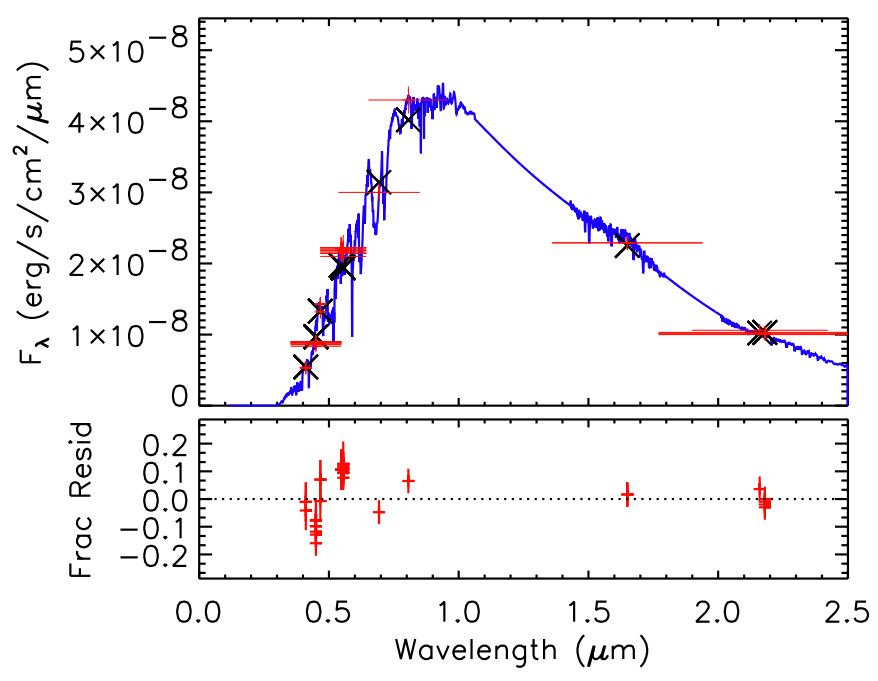

Figure 3. SED fit for G1 $15 \mathrm{~A}$ with a M2V spectral template (blue) from Pickles (1998) and photometry (red) from the literature. Filter bandpasses are indicated by the horizontal lengths of the red photometric measurements. The black " $\mathrm{X}$ " symbols show the template flux integrated over the filter bandpasses. Residuals between these integrated model and measured fluxes are in the lower panel. For details, see Section 2.3.2.

(A color version of this figure is available in the online journal.)

\subsubsection{Spectral Energy Distribution}

Similar to the procedure outlined in von Braun et al. (2012), we fitted the SED (Figure 3) based on the spectroscopic templates of Pickles (1998) to literature photometry published in Cowley et al. (1967), Johnson \& Morgan (1953), Upgren (1974), Niconov et al. (1957), Argue (1966), Johnson (1965), Erro (1971), Mermilliod (1986), Hauck \& Mermilliod (1998), Twarog (1980), Olsen (1993), Olson (1974), Cutri et al. (2003) and cataloged in Gezari et al. (1999). We did not include interstellar extinction from the SED fit due to the proximity of Gl $15 \mathrm{~A}$.

The SED fit produces a bolometric flux of $F_{\mathrm{bol}}=(5.42 \pm$ $0.04) \times 10^{-8} \mathrm{erg} \mathrm{cm}^{-2} \mathrm{~s}^{-1}$. The $\sim 1 \%$ fractional error accounts only for photometric and fitting uncertainties and does not include systematic uncertainties (e.g., see Section 2.2 of von Braun et al. 2014). Combining $F_{\text {bol }}$ with the distance, we derive a luminosity of $L=0.02173 \pm 0.00021 L_{\odot}$. We derive an effective temperature of $3563 \pm 11 \mathrm{~K}$ using the modified Stefan-Boltzmann Law $T_{\text {eff }}(\mathrm{K})=2341\left(F_{\text {bol }} / \theta_{\mathrm{LD}}^{2}\right)^{1 / 4}$ from von Braun et al. (2012).

Using the approach in von Braun et al. (2011b) and based on the equations of Jones \& Sleep (2010) we calculate the inner and outer boundaries of the habitable zone around Gl $15 \mathrm{~A}$ to be 0.14 and $0.29 \mathrm{AU}$, respectively. With a semi-major axis of $0.074 \mathrm{AU}$ (see Section 4), Gl $15 \mathrm{Ab}$ orbits interior to the habitable zone.

\subsection{Photometry from Fairborn Observatory}

We measured the brightness of G1 15 A over four observing seasons with the T12 $0.80 \mathrm{~m}$ automatic photometric telescope (APT), one of several automatic telescopes operated by Tennessee State University at Fairborn Observatory (Eaton et al. 2003). The APTs can detect short-term, low-amplitude brightness changes in solar-type stars resulting from rotational modulation in the visibility of active regions, such as starspots and plages (e.g., Henry et al. 1995b) and can also detect longerterm variations produced by the growth and decay of individual active regions and the occurrence of stellar magnetic cycles (e.g., Henry et al. 1995a; Hall et al. 2009). The TSU APTs can disprove the hypothesis that RV variations are caused by stellar activity, rather than planetary reflex motion (e.g., Henry et al. 2000a). Several cases of apparent periodic RV variations in solar-type stars induced by the presence of photospheric starspots have been discussed in the literature (e.g., Queloz et al. 2001; Paulson et al. 2004; Bonfils et al. 2007; Forveille et al. 2009). Photometry of planetary candidate host stars is also useful to search for transits of the planetary companions (e.g., Henry et al. 2000b; Sato et al. 2005; Gillon et al. 2007; Barbieri et al. 2007).

The T12 $0.80 \mathrm{~m}$ APT is equipped with a two-channel photometer that uses two EMI 9124QB bi-alkali photomultiplier tubes (PMTs) to make simultaneous measurements of one star in the Strömgren $b$ and $y$ passbands. We report differential measurements of the target star with respect to three comparison stars: HD $571(V=5.01, B-V=0.41)$, HD $818(V=6.63$, $B-V=0.40)$, and HD $1952(V=6.66, B-V=$ 0.41). The T12 APT is functionally identical to the T8 APT described in Henry (1999). All photometric measurements were made through a $45^{\prime \prime}$ focal-plane diaphragm, thus excluding the light from the two 11th magnitude visual companions Gl $15 \mathrm{~B}$ and $\mathrm{C}$. The observing sequence and conditions for rejecting photometry in nonphotometric conditions are described in Henry (1999).

During four consecutive observing seasons starting in 2008, the APT acquired 578 differential brightness measurements of Gl $15 \mathrm{~A}$. We combined the $b$ and $y$ differential magnitudes into $(b+y) / 2$ measurements, achieving typical single measurement precision of 1.5-2.0 mmag (Henry 1999). These measurements are plotted in the top panel of Figure 4 and have a standard deviation of $3.1 \mathrm{mmag}$, which is somewhat larger than measurement uncertainties.

In the 2011 observing season, we increased the cadence to several observations per night. A periodogram of these measurements (Figure 4, middle panel) shows Fourier power at a period of $43.82 \pm 0.56$ days. The phased photometry from this season is shown in the bottom panel of Figure 4. The APT photometry show no evidence for rotational modulation of spots at the orbital period to a limit of $\sim 0.1 \mathrm{mmag}$, thus supporting the interpretation that the 11.44 day RV signal is due to an orbiting planet (Section 4.1).

\section{KECK-HIRES DOPPLER MEASUREMENTS}

We measured the Doppler shift of each star-times-iodine HIRES spectrum (Section 2.1) using a modeling procedure descended from Butler et al. (1996) and described in Howard et al. (2011a). The velocity and corresponding uncertainty for each observation is based on separate measurements for $\sim 700$ spectral chunks each $2 \AA$ wide. Once the planet announced here emerged as a candidate in 2010 October, we increased the nightly cadence to three consecutive observations per night to reduce the Poisson noise from photon statistics. We calculated mean velocities for multiple observations in two hour intervals. The rms of these measurements is $3.21 \mathrm{~m} \mathrm{~s}^{-1}$. The relative RVs and simultaneous $S_{\mathrm{HK}}$ values are listed in Table 3 . The absolute RV of Gl 15 A relative to the solar system barycenter is $11.82 \pm 0.11 \mathrm{~km} \mathrm{~s}^{-1}$ (Chubak et al. 2012). The RVs in Table 3 are corrected for motion of Keck Observatory through the solar system (barycentric corrections) and secular acceleration, but not for any measured or assumed motion of G1 15 A from interactions with $\mathrm{Gl} 15 \mathrm{~B}$ or C. 

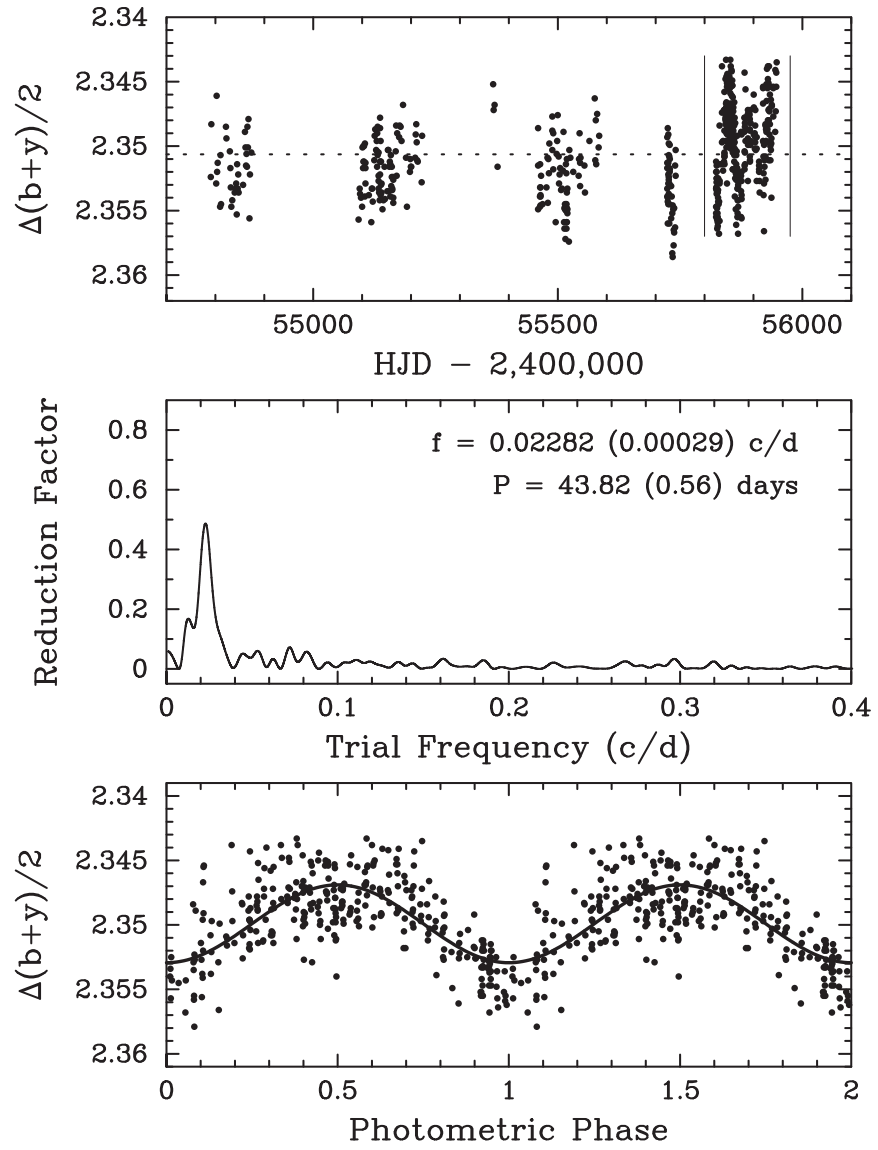

Figure 4. Top: Stromgren $(b+y) / 2$ differential magnitudes of G1 15 A plotted against heliocentric Julian Date for four observing seasons (2008-2011). The standard deviation of these normalized observations from their mean (dotted line) is $3.1 \mathrm{mmag}$. The vertical lines in the top panel set off the best covered and most coherent segment of the light curve that shows rotational modulation of spots. The two lower panels focus on this high cadence subset of the photometry. Middle: frequency spectrum of the high cadence photometry showing a peak corresponding to a period of $\sim 44$ days, which we interpret as the stellar rotation period. Bottom: photometry from the high cadence interval phased to a 44 day period. Two cycles are shown (with data repeated) along with a least-squared sine function fit having a peak-to-peak amplitude of 6 mmag.

Measurements made after the HIRES CCD upgrade in 2004 August suffer from smaller systematic errors. As described in the Section 4, when modeling the measurements we allowed for a zero-point offset between the 20 "pre-upgrade" and 97 "post-upgrade" RVs, as well as differing amounts of jitter.

\section{PLANET DETECTION AND ORBITAL MODEL}

We modeled the RV time series as a single planet in Keplerian orbit around the star G1 $15 \mathrm{~A}$. We searched for periodic signals by computing a Lomb-Scargle periodogram (Lomb 1976; Scargle 1982) of the RVs (Figure 5) and found a dominant peak at 11.44 days. We seeded single-planet Keplerian models with that period and a variety of other periods using the orbit fitting techniques described in Howard et al. (2010a) and the partially linearized, least-squares fitting procedure described in Wright \& Howard (2009). Our adopted model (Table 2) has an orbital period near 11.44 days. We also include a linear trend of $-0.26 \pm 0.09 \mathrm{~m} \mathrm{~s}^{-1} \mathrm{yr}^{-1}$, which is consistent with the mass and separation the G1 15 AB system (see Section 2). The best-fit circular, single-planet model is plotted in Figure 6.

We estimated orbital parameter uncertainties using a Markov Chain Monte Carlo (MCMC) method (Ford 2005, 2006) with

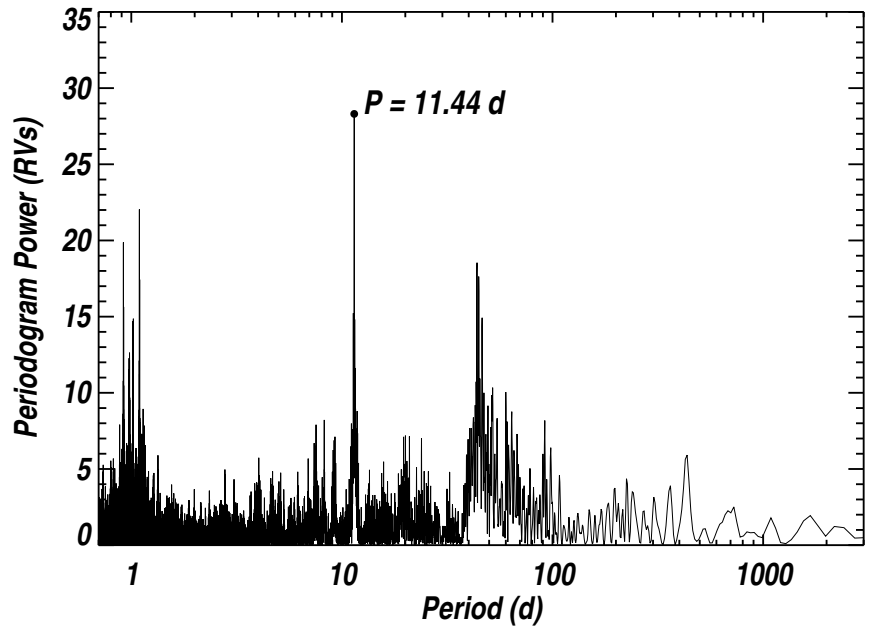

Figure 5. Lomb-Scargle periodogram of RV measurements of G1 15 A. The tall peak near $P=11.44$ days suggests a planet with that orbital period.

Table 2

Orbital Solutions for G1 $15 \mathrm{Ab}$

\begin{tabular}{|c|c|}
\hline Parameter & Value \\
\hline \multicolumn{2}{|l|}{ Circular Orbit Model (adopted) } \\
\hline$P$ (days) & $11.4433 \pm 0.0016$ \\
\hline$T_{c}{ }^{\mathrm{a}}(\mathrm{BJD}-2,440,000)$ & $15874.95 \pm 0.21$ \\
\hline$K\left(\mathrm{~m} \mathrm{~s}^{-1}\right)$ & $2.94 \pm 0.28$ \\
\hline$\gamma\left(\mathrm{m} \mathrm{s}^{-1}\right)$ & $2.47 \pm 0.88$ \\
\hline$d v / d t\left(\mathrm{~m} \mathrm{~s}^{-1} \mathrm{yr}^{-1}\right)$ & $-0.26 \pm 0.09$ \\
\hline$M \sin i\left(M_{\oplus}\right)$ & $5.35 \pm 0.75$ \\
\hline$a(\mathrm{AU})$ & $0.0717 \pm 0.0034$ \\
\hline rms to best-fit model, pre-upgrade RVs $\left(\mathrm{m} \mathrm{s}^{-1}\right)$ & 2.69 \\
\hline rms to best-fit model, post-upgrade RVs $\left(\mathrm{m} \mathrm{s}^{-1}\right)$ & 1.93 \\
\hline$\sigma_{\text {jit,pre }}\left(\mathrm{ms} \mathrm{s}^{-1}\right)$ & $2.56_{-0.48}^{+0.61}$ \\
\hline$\sigma_{\text {jit,post }}\left(\mathrm{m} \mathrm{s}^{-1}\right)$ & $1.88 \pm 0.16$ \\
\hline \multicolumn{2}{|l|}{ Eccentric Orbit Model } \\
\hline$P$ (days) & $11.4433 \pm 0.0017$ \\
\hline$T_{c}^{\mathrm{a}}(\mathrm{BJD}-2,440,000)$ & $15875.09 \pm 0.45$ \\
\hline$T_{p}^{\mathrm{b}}(\mathrm{BJD}-2,440,000)$ & $15876.6_{-4.7}^{+2.4}$ \\
\hline$e \cos \omega$ & $-0.04 \pm 0.11$ \\
\hline$e \sin \omega$ & $-0.01 \pm 0.09$ \\
\hline$e$ & $0.12_{-0.06}^{+0.08}$ \\
\hline$\omega(\mathrm{deg})$ & $186_{-110}^{+92}$ \\
\hline$K\left(\mathrm{~m} \mathrm{~s}^{-1}\right)$ & $2.93 \pm 0.29$ \\
\hline$\gamma\left(\mathrm{m} \mathrm{s}^{-1}\right)$ of relative RVs & $2.43 \pm 0.90$ \\
\hline$d v / d t\left(\mathrm{~m} \mathrm{~s}^{-1} \mathrm{yr}^{-1}\right)$ & $-0.27 \pm 0.09$ \\
\hline$M \sin i\left(M_{\oplus}\right)$ & $5.28 \pm 0.75$ \\
\hline$a(\mathrm{AU})$ & $0.0717 \pm 0.0036$ \\
\hline rms to best-fit model, pre-upgrade RVs $\left(\mathrm{m} \mathrm{s}^{-1}\right)$ & 2.70 \\
\hline rms to best-fit model, post-upgrade RVs $\left(\mathrm{m} \mathrm{s}^{-1}\right)$ & 1.93 \\
\hline$\sigma_{\mathrm{jit}, \mathrm{pre}}\left(\mathrm{m} \mathrm{s}^{-1}\right)$ & $2.54_{-0.48}^{+0.62}$ \\
\hline$\sigma_{\text {jit,post }}\left(\mathrm{ms}^{-1}\right)$ & $1.91 \pm 0.16$ \\
\hline
\end{tabular}

Notes.

a Time of inferior conjunction or time of transit if viewed edge-on.

b Time of periastron passage.

the Metropolis-Hastings algorithm (Metropolis et al. 1953; Hastings 1970) and a Gibbs sampler (Geman \& Geman 1984). We report the median, $84.1 \%$, and $15.9 \%$ levels of the marginalized posterior parameter distributions in Table 2. Instead of minimizing $\chi^{2}$, we (equivalently) maximized the logarithm of 


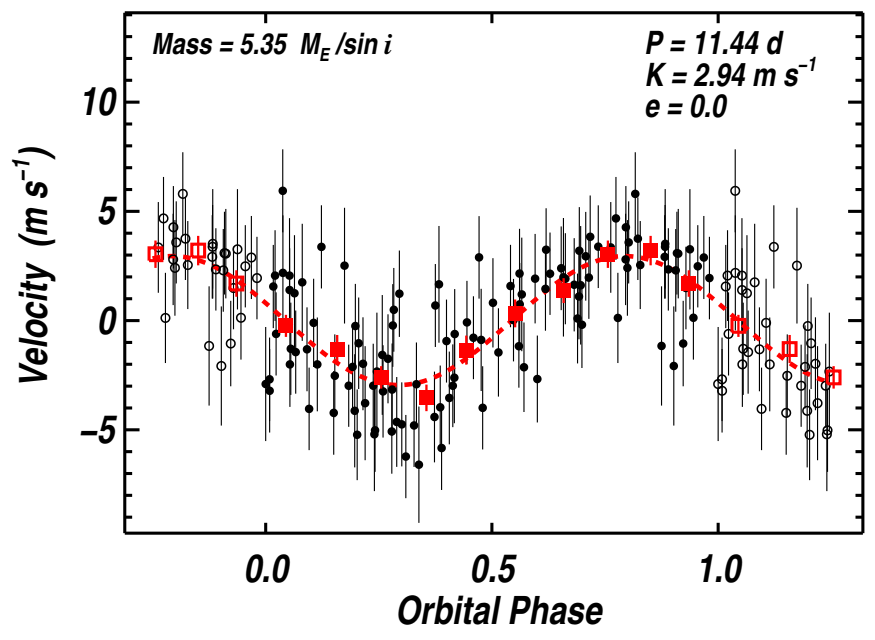

Figure 6. Single-planet model for the Keck-HIRES RVs of Gl $15 \mathrm{Ab}$. Filled black circles represent phased measurements while the open black circles represent the same velocities wrapped one orbital phase. The error bars show the quadrature sum of measurement uncertainties and jitter. Red squares show RVs binned in 0.1 phase increments and have an rms to the model of $0.62 \mathrm{~m} \mathrm{~s}^{-1}$. The best-fit circular orbital solution is shown as a dashed red line.

(A color version of this figure is available in the online journal.)

the likelihood,

$$
\begin{aligned}
\ln \mathcal{L}= & -\sum_{i=1}^{N_{\mathrm{pre}}} \frac{\left(v_{i}-v_{\mathrm{m}}\left(t_{i}\right)\right)^{2}}{2\left(\sigma_{i}^{2}+\sigma_{\mathrm{jit}, \mathrm{pre}}^{2}\right)}-\ln \sqrt{2 \pi\left(\sigma_{i}^{2}+\sigma_{\mathrm{jit}, \mathrm{pre}}^{2}\right)} \\
& -\sum_{i=1}^{N_{\mathrm{post}}} \frac{\left(v_{i}-v_{\mathrm{m}}\left(t_{i}\right)\right)^{2}}{2\left(\sigma_{i}^{2}+\sigma_{\mathrm{jit}, \mathrm{post}}^{2}\right)}-\ln \sqrt{2 \pi\left(\sigma_{i}^{2}+\sigma_{\mathrm{jit}, \mathrm{post}}^{2}\right)}
\end{aligned}
$$

where $v_{i}$ and $\sigma_{i}$ are the $i$ th velocity measurement and its associated measurement error from among the $N_{\text {pre }}$ and $N_{\text {post }}$ measurements acquired before and after the 2004 upgrade of HIRES; $v_{\mathrm{m}}\left(t_{i}\right)$ is the Keplerian model velocity at time $t_{i}$; $\sigma_{\mathrm{jit}, \text { pre }}$ and $\sigma_{\mathrm{jit}, \text { post }}$ are the jitter estimates of the pre- and postupgrade data sets. The first term in each sum represents the usual normalized sum of squared residuals $\left(\chi^{2}\right)$. Following Johnson et al. (2011), we allowed jitter to float in the MCMC analysis, as controlled by the second terms in each sum in Equation (1). Our model uses separate jitter parameters for the pre- and post-upgrade RV data sets. The jitter estimates for these data sets (see Table 2) show the improvement in HIRES measurement precision after the 2004 upgrade. We adopted a Gregory eccentricity prior (Gregory \& Fischer 2010) and noninformative priors on other parameters. The jump parameters of the MCMC model were orbital period $P$, a time of transit (or inferior conjunction) $T_{c}$, Doppler semi-amplitude $K$, Lagrangian parameters $e \cos \omega$ and $e \sin \omega$, RV zero-point $\gamma, \mathrm{RV}$ offset between pre- and post-upgrade data sets, linear RV trend $d v / d t$, and pre- and post-upgrade RV jitter terms $\sigma_{\text {jit,pre }}$ and $\sigma_{\mathrm{jit}, \text { post }}$. From these jump parameters we derived the remaining parameters listed in Table 2.

We justify the inclusion of a linear RV trend, $d v / d t$, as follows. For the circular planet model with a trend as a free parameter (Table 2, top), only $0.3 \%$ of the MCMC trials have $d v / d t>0$. Thus, a negative $\mathrm{RV}$ trend is preferred with approximately $3 \sigma$ significance. The trend is also physically well motivated by the distant orbit of the star Gl $15 \mathrm{~B}$, and is consistent with the $\sim 1 \mathrm{~m} \mathrm{~s}^{-1} \mathrm{yr}^{-1}$ expected amplitude (order of magnitude).
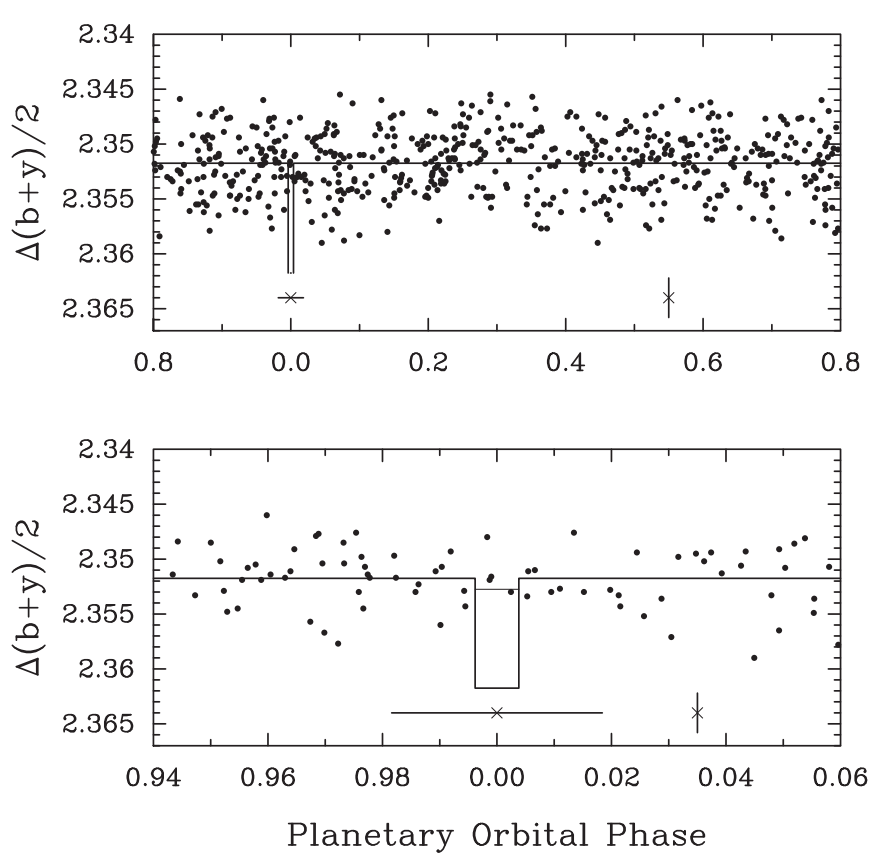

Figure 7. APT photometry from four observing seasons (2008-2011) phased to the best-fit orbital period (Table 2). The photometric means in the final three observing seasons were adjusted to remove yearly offsets. The top panel shows the entire orbital phase, while the bottom panel focuses on photometry near the phase of predicted transits. Photometry in the top panel shows no apparent modulation at the orbital period, which is consistent with our planetary interpretation of the RV modulation. Transit depths of 1 and 10 mmag are shown for a toy model with a transit duration of $2.1 \mathrm{hr}$ for an equatorial transit. The sparse photometry rules out transits if the planet's atmosphere is extremely bloated, but are insensitive to transits if the planet's atmosphere contributes minimally to its radius (see the text for details). The vertical bar shows the typical photometric error of $1.8 \mathrm{mmag}$. The transit time uncertainty for the circular orbit model is indicated by the horizontal line segment centered at phase 0.0 .

We considered circular and eccentric single-planet models. We adopted the circular orbit (Table 2, top) for two reasons. First, the posterior distributions for $e \cos \omega$ and $e \sin \omega$ are consistent with zero to within $1 \sigma$ in the floating eccentricity model. Second, the $\chi^{2}$ value (not reduced) of the best-fit eccentric model is only smaller than the value for the best-fit circular model by 0.3 , which fails to justify the addition of two model parameters. The eccentric model rules out $e>0.26$ with $95 \%$ confidence.

\subsection{Photometric Confirmation and Transit Search}

We searched for periodic variability of the APT photometry (Section 2.4) at the orbital period of 11.44 days, but found none. This nondetection strengthens the planetary interpretation for the 11.44 days RV signal. A least-squares sine fit to the photometry at the best-fit orbital period gives a semi-amplitude of $0.09 \pm 0.14 \mathrm{mmag}$. As shown in the top panel of Figure 7, this tight limit supports the hypothesis that the RV signal is due to stellar reflex motion from a planet in motion rather than spots.

Gl $15 \mathrm{Ab}$ has a $2 \%$ a priori probability of having an orbital inclination $i$ that gives rise to eclipses as seen from Earth. While this geometry is unlikely, it is instructive to consider the photometric detectability of transits. For plausible planets with radii 1.5-4.0 $R_{\oplus}$, corresponding to densities of $8-0.4 \mathrm{~g} \mathrm{~cm}^{-3}$, the transit depths are 1.4-10 mmag. If the planet has an Earthlike density, then $R_{p}=1.8 R_{\oplus}$, which gives a transit depth of 2.0 mmag. Equatorial transits will last $2.1 \mathrm{hr}$, as shown by the width of the box-shaped transit model in the bottom panel Figure 7. Our sparse APT photometry casts doubt on the presence of a 


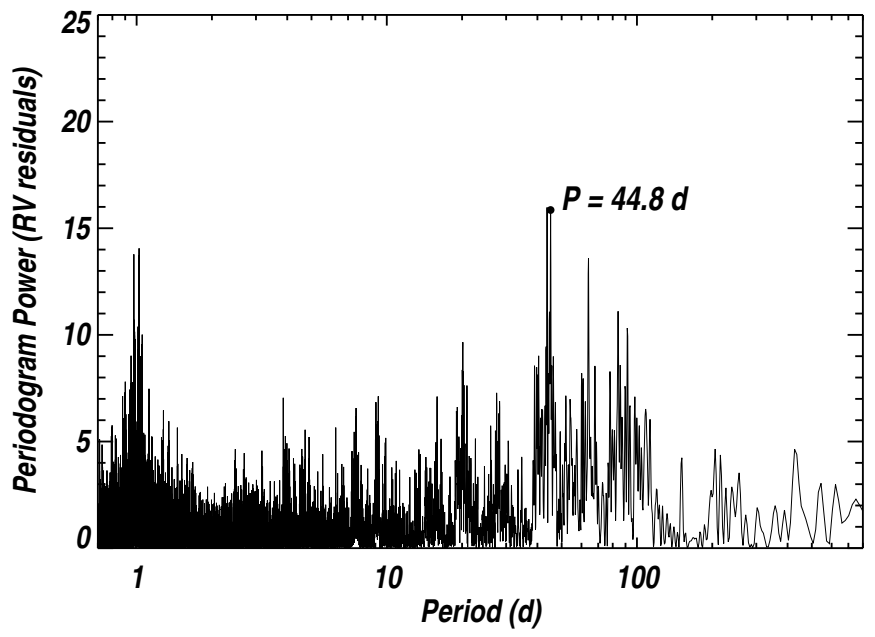

Figure 8. Lomb-Scargle periodogram of the RV residuals to the single-planet, circular orbit model for Gl $15 \mathrm{Ab}$. We interpret the peaks near 44 days as artifacts of stellar rotation because modulation at this period is also detected in $\mathrm{Ca}$ II $\mathrm{H}$ \& $\mathrm{K}$ lines and optical photometry.

very low density transiting planet. However, we refrain from making quantitative statements about the sizes of planets that can be excluded based on the current data. To date, only $\sim 4$ points are contained in the expected best-fit transit window. A dedicated photometric campaign from space or the ground could likely detect or exclude all of the planet sizes described above.

\subsection{Null Hypothesis Considered}

We considered the null hypothesis - that the observed RVs are the chance arrangement of random velocities masquerading as a coherent signal-by calculating false alarm probabilities (FAPs) and Bayesian Information Criteria (BIC). Using the method described in Howard et al. (2010a), we computed the improvement in $\Delta \chi^{2}$ from a constant velocity model to a Keplerian model for $10^{3}$ scrambled data sets. We allowed for eccentric single-planet orbital solutions in the scrambled data sets. We found that no scrambled data set had a larger $\Delta \chi^{2}$ value than the measured velocities did, implying an FAP of $<0.001$.

As an additional check, we assessed statistical significance by computing the BIC (Schwarz 1978; Liddle 2004) for the single planet circular model (Table 2, top) and the null hypothesis, a model with an RV trend varying linearly with time. The BIC is defined as $\chi_{\min }^{2}+k \ln N_{\text {obs }}$, where $\chi_{\min }^{2}$ is the unreduced $\chi^{2}$ value of the best-fitting model having $k$ degrees of freedom. $N_{\text {obs }}=$ 117 is the number of RV measurements. Differences of 2-6, $6-10,>10$ between BIC values for the two models indicates that there is positive, strong, and very strong evidence for the more complex model (Kass \& Raftery 1995). The single planet model (Table 2) is strongly supported over the null hypothesis with $\Delta \mathrm{BIC}=24$.

\subsection{A Second RV Signal}

In addition to the single-planet model presented above, we also considered two-planet Keplerian models. The small residuals to the one-planet fit constrain the family of possible additional planets to those with small Doppler amplitudes-low mass and/or distant. We computed a periodogram of the RV residuals (Figure 8 ) to the single-planet fit and found several periods with considerable power in the range $\sim 30-120$ days, with the two largest peaks near 44 days. These peaks correspond to Doppler signals with $\sim 1-2 \mathrm{~m} \mathrm{~s}^{-1}$ semiamplitudes and represent possible second planets with masses in the range $\sim 2-10 M_{\oplus}$.
We considered two-planet orbital solutions with $P_{b}$ seeded with the best-fit value from the single-planet model and $P_{c}$ seeded with peaks in the residual periodogram. Fits with $P_{c}$ seeded with periods corresponding to either of two tallest peaks in Figure 8 yield the most statistically significant two-planet fits. If real, this signal would represent a $5 M_{\oplus}$ planet in a $0.18 \mathrm{AU}$ orbit within the classically defined habitable zone. The signal (at either of the two periods near 44 days) has an amplitude of $1.8 \mathrm{~m} \mathrm{~s}^{-1}$.

However, we do not interpret the 44 day RV signal as a planet. The broad periodogram power in the $\sim 30-120$ day range, with two closely spaced peaks near $44 \mathrm{~d}$, suggests that it is due to rotationally modulated spots. Such spots appear and disappear on timescales of weeks and months on a range of differentially rotating stellar latitudes, injecting a quasi-coherent noise in the RV time series. In contrast, an orbiting planet creates a coherent RV signature. The spot interpretation is strengthened with the detection of periodic signals in the photometry (Section 2.4) and $\mathrm{Ca}$ II $\mathrm{H} \& \mathrm{~K}$ line strengths (Section 2.1), also with $\sim 44$ day periods. We measured the correlation between the RV residuals to the one-planet fit and the simultaneous $S_{\mathrm{HK}}$ values. The Pearson correlation coefficient of $r=+0.41$ demonstrates a modest correlation between these quantities. In comparison, the RVs in Table 3 (before subtracting the single-planet fit) are less correlated with the $S_{\mathrm{HK}}$ values, with $r=+0.24$. We conclude that the $\sim 44$ days RV signal is mostly likely not due to an orbiting planet.

As an additional check, we computed "running periodograms" (Howard et al. 2011a) of the 11.4 day and $~ 44$ day signals. This diagnostic tests for coherent signals in the RV time series by computing the increase in Lomb-Scargle periodogram power near a trial period as additional measurements are taken. The 11.4 day signal rises nearly monotonically in the time series, suggesting a dynamical origin and supporting the planetary interpretation. In contrast, the $\sim 44$ day signal waxes and wanes as additional measurements are added to the time series, suggesting an incoherent source such as rotationally modulated spots.

\section{SUMMARY AND DISCUSSION}

We announce the existence of a low-mass planet orbiting the star G1 15 A, an M2 dwarf 3.6 pc from Earth. G1 15 Ab has a minimum mass $5.28 \pm 0.75 M_{\oplus}$ and orbits with a period of $11.4433 \pm 0.0017$ days. The orbital eccentricity is consistent with zero and the planet has an equilibrium temperature of $390 \mathrm{~K}$ for a Bond albedo $A=0.75$ and $550 \mathrm{~K}$ for $A=0$.

In addition, we detected a second RV signal with a period of 44 days and an amplitude of $1.8 \mathrm{~m} \mathrm{~s}^{-1}$. Because we also detected photometric and chromospheric modulation with the same period, we interpret this signal as rotational modulation of spots. We will continue to monitor Gl 15 A to verify that the second signal is truly incoherent, as spots should be, and to hunt for additional low-mass planets. Our analysis of this second signal shows how challenging the RV detection of Earth-mass planets in the habitable zones of early $M$ dwarfs will be. Such planets will have periods of $\sim 1-2$ months and Doppler amplitudes of $\sim 0.3-0.4 \mathrm{~m} \mathrm{~s}^{-1}$, i.e., with similar periods to the spot signal above, but five times smaller in amplitude. Detecting such signals will be quite challenging, but may be feasible for high-precision Doppler spectrometers with nearly nightly observational coverage. The high cadence not only improves the (naïve) sensitivity as $\sqrt{N_{\text {obs }}}$, it allows the observer to trace out the anomalous RV signature of stellar spots and 
Table 3

Relative Radial Velocities for Gl 15 A

\begin{tabular}{|c|c|c|c|}
\hline BJD - 2,440,000 & $\begin{array}{l}\text { Radial Velocity } \\
\qquad\left(\mathrm{m} \mathrm{s}^{-1}\right)\end{array}$ & $\begin{array}{l}\text { Uncertainty } \\
\left(\mathrm{m} \mathrm{s}^{-1}\right)\end{array}$ & $S_{\mathrm{HK}}$ \\
\hline 10461.77113 & -2.79 & 1.09 & 0.6000 \\
\hline 10716.03838 & -3.68 & 1.09 & 0.6270 \\
\hline 11044.04091 & 3.60 & 0.81 & 0.6650 \\
\hline 11071.01567 & -5.12 & 1.07 & 0.5980 \\
\hline 11368.04395 & -4.06 & 1.04 & 0.5700 \\
\hline 11412.00051 & 2.25 & 1.15 & 0.5690 \\
\hline 11438.83506 & 1.73 & 1.23 & 0.7620 \\
\hline 11552.75997 & -6.53 & 1.21 & 0.5040 \\
\hline 11704.12495 & 1.26 & 1.06 & 0.5640 \\
\hline 11882.73441 & 2.56 & 1.18 & 0.6340 \\
\hline 12063.12014 & 3.31 & 1.40 & 0.6000 \\
\hline 12097.04192 & -2.04 & 1.35 & 0.5680 \\
\hline 12099.08799 & 1.79 & 1.25 & 0.5660 \\
\hline 12129.01691 & 1.65 & 1.32 & 0.5630 \\
\hline 12133.09619 & 2.10 & 1.14 & 0.5500 \\
\hline 12487.96446 & 1.27 & 1.20 & 0.5850 \\
\hline 12535.95822 & -1.55 & 1.38 & 0.5760 \\
\hline 12574.86983 & 2.00 & 1.32 & 0.5310 \\
\hline 12829.10405 & -1.15 & 1.35 & 0.5360 \\
\hline 12924.89439 & -2.33 & 1.28 & $\ldots$ \\
\hline 13238.98277 & 3.49 & 0.84 & 0.5840 \\
\hline 13302.80391 & -1.46 & 0.93 & 0.4880 \\
\hline 13338.81208 & -2.33 & 1.03 & 0.5460 \\
\hline 13547.11252 & 3.52 & 0.57 & 0.5400 \\
\hline 13548.11449 & 3.22 & 0.92 & 0.5300 \\
\hline 13549.12933 & 4.55 & 0.53 & 0.5280 \\
\hline 13550.12107 & 3.65 & 0.61 & 0.5330 \\
\hline 13551.10142 & 3.16 & 0.58 & 0.5575 \\
\hline 13552.06885 & 1.68 & 0.62 & 0.5230 \\
\hline 13571.07965 & 2.24 & 0.54 & 0.5260 \\
\hline 13723.72563 & -1.74 & 0.71 & 0.5385 \\
\hline 13928.02017 & 2.56 & 0.55 & 0.5720 \\
\hline 13981.94154 & 1.71 & 0.62 & 0.5480 \\
\hline 14085.85430 & 0.06 & 0.80 & 0.5255 \\
\hline 14339.08172 & 2.78 & 0.88 & 0.6430 \\
\hline 14340.04292 & 3.31 & 0.95 & 0.6390 \\
\hline 14398.90264 & -1.05 & 1.09 & 0.5990 \\
\hline 14429.88240 & 3.59 & 1.02 & 0.6160 \\
\hline 14667.99675 & -1.91 & 0.96 & 0.5600 \\
\hline 14672.02334 & -0.82 & 0.74 & 0.5255 \\
\hline 14673.00572 & -2.46 & 0.61 & 0.5175 \\
\hline 14674.11984 & 0.12 & 0.89 & 0.5420 \\
\hline 14676.09669 & -4.85 & 0.68 & 0.5220 \\
\hline 14689.13119 & -0.39 & 0.98 & 0.6190 \\
\hline 14690.09869 & 1.04 & 0.95 & 0.5370 \\
\hline 14721.03229 & -0.81 & 1.05 & 0.5550 \\
\hline 14778.86564 & -3.02 & 1.09 & 0.4860 \\
\hline 14807.88673 & 3.02 & 1.05 & 0.5410 \\
\hline 15134.90869 & -4.21 & 1.02 & 0.5290 \\
\hline 15412.09294 & 2.11 & 0.96 & 0.4720 \\
\hline 15434.05192 & -1.27 & 0.90 & 0.5200 \\
\hline 15435.03590 & -2.48 & 0.82 & 0.4940 \\
\hline 15436.05321 & 0.29 & 0.97 & 0.4860 \\
\hline 15437.07327 & 0.31 & 0.99 & 0.5260 \\
\hline 15470.07431 & 2.11 & 1.03 & 0.5210 \\
\hline 15490.02622 & -3.35 & 1.11 & 0.4420 \\
\hline 15528.86024 & 2.60 & 1.23 & 0.4850 \\
\hline 15542.83596 & 2.25 & 1.02 & 0.5070 \\
\hline 15545.79628 & -2.97 & 1.08 & 0.4880 \\
\hline 15584.73104 & 1.83 & 1.13 & 0.5180 \\
\hline 15613.71949 & -1.79 & 1.12 & 0.4840 \\
\hline 15704.11022 & -1.82 & 1.15 & 0.5090 \\
\hline 15705.11833 & -5.05 & 1.02 & 0.5210 \\
\hline 15706.11861 & -4.46 & 0.97 & 0.5110 \\
\hline 15723.10704 & 4.86 & 0.57 & 0.4863 \\
\hline
\end{tabular}

Table 3

(Continued)

\begin{tabular}{|c|c|c|c|}
\hline BJD - 2,440,000 & $\begin{array}{l}\text { Radial Velocity } \\
\qquad\left(\mathrm{m} \mathrm{s}^{-1}\right)\end{array}$ & $\begin{array}{l}\text { Uncertainty } \\
\left(\mathrm{m} \mathrm{s}^{-1}\right)\end{array}$ & $S_{\mathrm{HK}}$ \\
\hline 15726.12358 & 6.12 & 0.57 & 0.4990 \\
\hline 15727.10118 & 3.55 & 0.58 & 0.5210 \\
\hline 15729.07256 & 1.41 & 0.79 & 0.5375 \\
\hline 15731.08821 & 3.07 & 0.53 & 0.5337 \\
\hline 15732.11181 & 2.33 & 0.95 & 0.5420 \\
\hline 15734.10300 & 3.56 & 0.55 & 0.5540 \\
\hline 15735.10478 & 3.93 & 0.54 & 0.5353 \\
\hline 15736.09232 & 3.27 & 0.56 & 0.5317 \\
\hline 15752.12244 & -6.05 & 0.59 & 0.4737 \\
\hline 15753.02718 & -5.65 & 0.64 & 0.4593 \\
\hline 15760.12146 & -3.03 & 0.61 & 0.4450 \\
\hline 15761.11957 & -3.86 & 0.54 & 0.4463 \\
\hline 15762.12115 & -2.80 & 0.50 & 0.4370 \\
\hline 15770.11306 & 3.10 & 0.60 & 0.4967 \\
\hline 15770.94986 & 2.66 & 0.52 & 0.5213 \\
\hline 15782.09409 & 1.67 & 0.54 & 0.5440 \\
\hline 15783.10066 & 1.74 & 0.59 & 0.5480 \\
\hline 15786.12265 & -0.05 & 0.55 & 0.5343 \\
\hline 15787.99817 & 0.10 & 0.58 & 0.5217 \\
\hline 15789.09994 & 1.76 & 0.52 & 0.5203 \\
\hline 15790.09912 & 2.32 & 0.53 & 0.5370 \\
\hline 15791.11511 & 4.01 & 0.55 & 0.5150 \\
\hline 15792.08892 & 3.76 & 0.51 & 0.5070 \\
\hline 15793.09169 & 2.52 & 0.52 & 0.4887 \\
\hline 15794.13095 & 2.12 & 0.53 & 0.4933 \\
\hline 15795.11032 & -1.26 & 0.58 & 0.4870 \\
\hline 15796.09957 & -2.35 & 0.56 & 0.4857 \\
\hline 15797.11759 & -4.85 & 0.57 & 0.4880 \\
\hline 15798.10397 & -4.62 & 0.57 & 0.5193 \\
\hline 15799.08373 & -2.81 & 0.56 & 0.4947 \\
\hline 15806.83968 & -1.14 & 0.64 & 0.4497 \\
\hline 15808.06252 & -0.07 & 0.66 & 0.4370 \\
\hline 15809.03505 & 0.67 & 0.63 & 0.4497 \\
\hline 15810.08551 & 0.87 & 0.57 & 0.4433 \\
\hline 15811.04909 & -0.61 & 0.57 & 0.4670 \\
\hline 15812.06251 & 0.20 & 0.53 & 0.4620 \\
\hline 15815.14191 & 5.98 & 0.59 & 0.4940 \\
\hline 15841.84563 & -4.05 & 0.62 & 0.4930 \\
\hline 15842.84489 & -2.81 & 0.65 & 0.4730 \\
\hline 15843.93703 & -2.74 & 0.61 & 0.4727 \\
\hline 15850.94268 & 0.30 & 0.62 & 0.4707 \\
\hline 15851.83105 & -0.44 & 0.58 & 0.4373 \\
\hline 15853.76621 & -1.94 & 0.58 & 0.4367 \\
\hline 15870.93426 & 1.28 & 0.74 & 0.5607 \\
\hline 15877.89696 & -4.58 & 0.65 & 0.5207 \\
\hline 15878.86899 & -3.80 & 0.61 & 0.5217 \\
\hline 15879.94263 & -3.82 & 0.59 & 0.5037 \\
\hline 15880.85881 & -1.00 & 0.57 & 0.5250 \\
\hline 15901.91394 & -0.77 & 0.57 & 0.4713 \\
\hline 15902.79143 & -0.71 & 1.48 & 0.4940 \\
\hline 15903.76626 & 0.92 & 0.56 & 0.4703 \\
\hline 15904.81574 & 2.57 & 0.63 & 0.4743 \\
\hline
\end{tabular}

potentially model and subtract it (e.g., Dumusque et al. 2012). Contemporaneous photometry will also aid in the false positive vetting for such future searches.

Statistical studies of the Kepler planet catalog suggest that small planets like Gl $15 \mathrm{Ab}$ are abundant (Howard et al. 2012; Howard 2013) and that multi-planet systems are common (Lissauer et al. 2011; Fang \& Margot 2012). We plan continued $\mathrm{RV}$ monitoring to search for such additional planets in this system. Given the distance of only $3.6 \mathrm{pc}$, we urge high-contrast imaging and astrometry by next generation surveys. 
We thank the many observers who contributed to the measurements reported here. We gratefully acknowledge the efforts and dedication of the Keck Observatory staff, especially Scott Dahm, Greg Doppman, Hien Tran, and Grant Hill for support of HIRES and Greg Wirth for support of remote observing. We thank Kevin Apps, Andrew Mann, Evan Sinukoff, and Calla Howard for helpful discussions. We are grateful to the time assignment committees of the University of Hawaii, the University of California, and NASA for their generous allocations of observing time. Without their long-term commitment to RV monitoring, this planet would likely remain unknown. We acknowledge R. Paul Butler and S.S. Vogt for many years of contributing to the data presented here. A.W.H. acknowledges NASA grant NNX12AJ23G. G.W.H. acknowledges support from NASA, NSF, Tennessee State University, and the State of Tennessee through its Centers of Excellence program. J.A.J. gratefully acknowledges support from generous grants from the David \& Lucile Packard and Alfred P. Sloan Foundations. This work made use of the SIMBAD database (operated at CDS, Strasbourg, France), NASA's Astrophysics Data System Bibliographic Services, and the NASA Star and Exoplanet Database (NStED). Finally, the authors wish to extend special thanks to those of Hawai'ian ancestry on whose sacred mountain of Mauna Kea we are privileged to be guests. Without their generous hospitality, the Keck observations presented herein would not have been possible.

\section{REFERENCES}

Allard, F., Homeier, D., \& Freytag, B. 2012, RSPTA, 370, 2765 Argue, A. N. 1966, MNRAS, 133, 475

Bailey, J., Butler, R. P., Tinney, C. G., et al. 2009, ApJ, 690, 743

Baraffe, I., Chabrier, G., Allard, F., \& Hauschildt, P. H. 1998, A\&A, 337,403

Barbieri, M., Alonso, R., Laughlin, G., et al. 2007, A\&A, 476, L13

Berger, D. H., Gies, D. R., McAlister, H. A., et al. 2006, ApJ, 644, 475

Bonfils, X., Mayor, M., Delfosse, X., et al. 2007, A\&A, 474, 293

Boyajian, T. S., von Braun, K., van Belle, G., et al. 2012, ApJ, 757, 112

Butler, R. P., Marcy, G. W., Williams, E., et al. 1996, PASP, 108, 500

Chubak, C., Marcy, G., Fischer, D. A., et al. 2012, arXiv:1207.6212

Claret, A. 2000, A\&A, 363, 1081

Covey, K. R., Lada, C. J., Román-Zúñiga, C., et al. 2010, ApJ, 722, 971

Cowley, A. P., Hiltner, W. A., \& Witt, A. N. 1967, AJ, 72, 1334

Cushing, M. C., Rayner, J. T., \& Vacca, W. D. 2005, ApJ, 623, 1115

Cushing, M. C., Vacca, W. D., \& Rayner, J. T. 2004, PASP, 116, 362

Cutri, R. M., Skrutskie, M. F., van Dyk, S., et al. 2003, yCat, 2246, 0

Delfosse, X., Forveille, T., Mayor, M., et al. 1998, A\&A, 338, L67

Delfosse, X., Forveille, T., Ségransan, D., et al. 2000, A\&A, 364, 217

Dotter, A., Chaboyer, B., Jevremović, D., et al. 2008, ApJS, 178, 89

Dumusque, X., Pepe, F., Lovis, C., et al. 2012, Natur, 491, 207

Eaton, J. A., Henry, G. W., \& Fekel, F. C. 2003, in The Future of Small Telescopes in the New Millennium, Vol. II, The Telescopes We Use, ed. T. D. Oswalt (Astrophysics and Space Science Library, Vol. 288; Dordrecht: Kluwer), 189 Erro, B. I. 1971, BITon, 6, 143

Fang, J., \& Margot, J.-L. 2012, ApJ, 761, 92

Feiden, G. A., Chaboyer, B., \& Dotter, A. 2011, ApJL, 740, L25

Ford, E. B. 2005, AJ, 129, 1706

Ford, E. B. 2006, ApJ, 642, 505

Forveille, T., Bonfils, X., Delfosse, X., et al. 2009, A\&A, 493, 645

Fowler, A. M., \& Gatley, I. 1990, ApJL, 353, L33

Gautier, T. N., III, Rieke, G. H., Stansberry, J., et al. 2007, ApJ, 667, 527

Geman, S., \& Geman, D. 1984, ITPAM, 6, 721

Gezari, D. Y., Pitts, P. S., \& Schmitz, M. 1999, yCat, 2225, 0

Gillon, M., Pont, F., Demory, B., et al. 2007, A\&A, 472, L13

Gregory, P. C., \& Fischer, D. A. 2010, MNRAS, 403, 731

Hall, J. C., Henry, G. W., Lockwood, G. W., Skiff, B. A., \& Saar, S. H. 2009, AJ, 138,312

Hastings, W. K. 1970, Biometrika, 57, 97

Hatzes, A. P., Cochran, W. D., McArthur, B., et al. 2000, ApJL, 544, L145

Hauck, B., \& Mermilliod, M. 1998, A\&AS, 129, 431
Henry, G. W. 1999, PASP, 111, 845

Henry, G. W., Baliunas, S. L., Donahue, R. A., Fekel, F. C., \& Soon, W. 2000a, ApJ, 531, 415

Henry, G. W., Eaton, J. A., Hamer, J., \& Hall, D. S. 1995a, ApJS, 97, 513

Henry, G. W., Fekel, F. C., \& Hall, D. S. 1995b, AJ, 110, 2926

Henry, G. W., Marcy, G. W., Butler, R. P., \& Vogt, S. S. 2000b, ApJL, 529, L41

Henry, T. J., Jao, W.-C., Subasavage, J. P., et al. 2006, AJ, 132, 2360

Herter, T. L., Henderson, C. P., Wilson, J. C., et al. 2008, Proc. SPIE, 7014, 70140X

Houdebine, E. R. 2010, MNRAS, 407, 1657

Howard, A. W. 2013, Sci, 340, 572

Howard, A. W., Johnson, J. A., Marcy, G. W., et al. 2010a, ApJ, 721, 1467

Howard, A. W., Johnson, J. A., Marcy, G. W., et al. 2009, ApJ, 696, 75

Howard, A. W., Johnson, J. A., Marcy, G. W., et al. 2011a, ApJ, 726, 73

Howard, A. W., Johnson, J. A., Marcy, G. W., et al. 2011b, ApJ, 730, 10

Howard, A. W., Johnson, J. A., Marcy, G. W., et al. 2010b, Sci, 330, 653

Howard, A. W., Marcy, G. W., Bryson, S. T., et al. 2012, ApJS, 201, 15

Isaacson, H., \& Fischer, D. 2010, ApJ, 725, 875

Jao, W.-C., Henry, T. J., Subasavage, J. P., et al. 2005, AJ, 129, 1954

Johnson, H. L. 1965, ApJ, 141, 170

Johnson, H. L., \& Morgan, W. W. 1953, ApJ, 117, 313

Johnson, J. A., \& Apps, K. 2009, ApJ, 699, 933

Johnson, J. A., Clanton, C., Howard, A. W., et al. 2011, ApJS, 197, 26

Johnson, J. A., Gazak, J. Z., Apps, K., et al. 2012, AJ, 143, 111

Jones, B. W., \& Sleep, P. N. 2010, MNRAS, 407, 1259

Kass, R., \& Raftery, A. 1995, J. Am. Stat. Assoc., 90, 773

Leggett, S. K. 1992, ApJS, 82, 351

Liddle, A. R. 2004, MNRAS, 351, L49

Lippincott, S. L. 1972, AJ, 77, 165

Lissauer, J. J., Ragozzine, D., Fabrycky, D. C., et al. 2011, ApJS, 197, 8

Lomb, N. R. 1976, Ap\&SS, 39, 447

Marcy, G. W., \& Butler, R. P. 1992, PASP, 104, 270

Marcy, G. W., Butler, R. P., Vogt, S. S., Fischer, D., \& Lissauer, J. J. 1998, ApJL, $505, \mathrm{~L} 147$

Mayor, M., Bonfils, X., Forveille, T., et al. 2009, A\&A, 507, 487

Mermilliod, J.-C. 1986, Catalogue of Eggen's UBV Data, 0, 0

Metropolis, N., Rosenbluth, A. W., Rosenbluth, M. N., Teller, A. H., \& Teller, E. 1953, JChPh, 21, 1087

Niconov, V. B., Nekrasova, S. V., Polosuina, N. S., Rachkouvsky, N. D., \& Chuvajev, W. K. 1957, IzKry, 17, 42

Olsen, E. H. 1993, A\&AS, 102, 89

Olson, E. C. 1974, AJ, 79, 1424

Paulson, D. B., Saar, S. H., Cochran, W. D., \& Henry, G. W. 2004, AJ, 127,1644

Pepe, F., Lovis, C., Ségransan, D., et al. 2011, A\&A, 534, A58

Pickles, A. J. 1998, PASP, 110, 863

Queloz, D., Henry, G. W., Sivan, J. P., et al. 2001, A\&A, 379, 279

Rayner, J. T., Cushing, M. C., \& Vacca, W. D. 2009, ApJS, 185, 289

Reid, I. N., Hawley, S. L., \& Gizis, J. E. 1995, AJ, 110, 1838

Rivera, E. J., Laughlin, G., Butler, R. P., et al. 2010, ApJ, 719, 890

Rojas-Ayala, B., Covey, K. R., Muirhead, P. S., \& Lloyd, J. P. 2010, ApJL, 720, L113

Rojas-Ayala, B., Covey, K. R., Muirhead, P. S., \& Lloyd, J. P. 2012, ApJ, 748,93

Sato, B., Fischer, D. A., Henry, G. W., et al. 2005, ApJ, 633, 465

Scargle, J. D. 1982, ApJ, 263, 835

Schwarz, G. 1978, AnSta, 6, 461

ten Brummelaar, T. A., McAlister, H. A., Ridgway, S. T., et al. 2005, ApJ, 628,453

Twarog, B. A. 1980, ApJS, 44, 1

Upgren, A. R. 1974, PASP, 86, 294

Vacca, W. D., Cushing, M. C., \& Rayner, J. T. 2003, PASP, 115, 389

Valenti, J. A., Butler, R. P., \& Marcy, G. W. 1995, PASP, 107, 966

Valenti, J. A., \& Fischer, D. A. 2005, ApJS, 159, 141

Valenti, J. A., \& Piskunov, N. 1996, A\&AS, 118, 595

van Belle, G. T., \& von Braun, K. 2009, ApJ, 694, 1085

van Buren, D., Brundage, M., Ressler, M., \& Terebey, S. 1998, AJ, 116, 1992

van Leeuwen, F. 2007, A\&A, 474, 653

Vogt, S. S., Allen, S. L., Bigelow, B. C., et al. 1994, Proc. SPIE, 2198, 362

von Braun, K., Boyajian, T. S., Kane, S. R., et al. 2012, ApJ, 753, 171

von Braun, K., Boyajian, T. S., Kane, S. R., et al. 2011a, ApJL, 729, L26

von Braun, K., Boyajian, T. S., ten Brummelaar, T. A., et al. 2011b, ApJ,

740,49

von Braun, K., Boyajian, T. S., van Belle, G. T., et al. 2014, MNRAS, 438, 2413

Wilson, J. C., Henderson, C. P., Herter, T. L., et al. 2004, Proc. SPIE, 5492, 1295

Wright, J. T., \& Howard, A. W. 2009, ApJS, 182, 205 